\title{
Molecular Mechanisms of Notopterygii Rhizoma Et Radix for the Treatment of Arrhythmia Based on Network Pharmacology
}

\section{PL Wei ( $\sim 1294072932 @ q q . c o m)$}

Beijing Hospital of Traditional Chinese Medicine

Yifei Qi

China Academy of Chinese Medical Sciences Xiyuan Hospital

\section{Yupei Tan}

Beijing University of Chinese Medicine

\section{Dehuai Long}

Beijing Hospital of Traditional Chinese Medicine

\section{Wenlong Xing}

Beijing Hospital of Traditional Chinese Medicine

\section{Xiang Li}

Beijing Hospital of Traditional Chinese Medicine

\section{Juju Shang}

Beijing Hospital of Traditional Chinese Medicine

Hongxu Liu

Beijing Hospital of Traditional Chinese Medicine

\section{Research}

Keywords: Notopterygii Rhizoma Et Radix, Arrhythmia, Molecular Mechanisms, Network analysis, Molecular docking

Posted Date: November 23rd, 2021

DOI: https://doi.org/10.21203/rs.3.rs-1047765/v1

License: (c) (i) This work is licensed under a Creative Commons Attribution 4.0 International License. Read Full License 


\section{Abstract}

Background Many experiments showed that Notopterygii Rhizoma Et Radix (NRR) can resist arrhythmia, but the mechanism of its action has not clear. Here, we investigated the possible mechanisms of NRR by network pharmacology and molecular docking and verified them experimentally.

Methods Active componds and targets of NRR were retrieved by the Traditional Chinese Medicine Systems Pharmacology (TCMSP) Database andAnalysis Platform, SymMap, and the Encyclopedia of Traditional Chinese Medicine (ETCM) databases. Arrhythmia-related targets were acquired from Comparative Toxicogenomics Database (CTD) and GeneCards databases. Overlapping targets of NRR associated with arrhythmia were acquired via Venn diagram. DAVID was applied for GO and KEGG pathway analyses. Cytoscape software and its plug-in were used for PPI network construction, module division and hub nodes screening. AutoDock Vina and qRT-PCR were carried out for validation.

Results The 21 active compounds and 57 targets were obtained. Of these, coumarin was the predominant category including 15 components and 31 targets. The 5 key targets of NRR in treating arrhythmia, and these targets are involved in the apoptotic process, extrinsic apoptotic signaling pathway in absence of ligand, endopeptidase activity involved in apoptotic process by cytochrome $\mathrm{c}$. The main pathways are p53 signaling pathway, Hepatitis B and Apoptosis. The results of molecular docking and qRT-PCR display good effect on hub node regulation in NRR treatment.

Conclusion NRR plays an important role in anti-apoptotic mediated by modulating p53 signaling pathway, which may provide insight into future research and clinical applications in arrhythmia therapy.

\section{Background}

Ventricular arrhythmias are a major cause of morbidity and mortality in patients with heart disease[1]. It can be manifested as asymptomatic premature ventricular contractions (PVCs) or non-sustained ventricular tachycardia (VT), and its symptoms are arrhythmia, or continuous ventricular tachycardia, with mild symptoms to complete hemodynamics collapse[2-3]. Conventional treatments for ventricular arrhythmia include antiarrhythmic drugs, catheter ablation or embedded cardioversion defibrillator; However, the adverse effects of defibrillation on physical and emotional and the huge toxicity and limited efficacy of anti-arrhythmia limit its application[3]. Moreover, quantifiable and molecular-based systems biology approaches are proposed [4].

High-quality datasets, combined with the results of genetic, genomic or other molecular analyses and complementing the work of experimental systems, ideally of human origin, can model functional consequences[5-8], which may characterize the potentially deleterious changes or interventions that affect disease phenotypes[9-10]. Network pharmacology could integrate these datasets through complicated interaction network[11-12], and elucidate the mechanisms of interventions from the system level. 
Traditional Chinese Medicine (TCM) has been widely used since ancient civilization, and it has become one of the main forms of alternative medicine in East Asia, North America, and Europe[13]. Chinese herbal medicine acts on multiple targets through multiple pathways, which is very similar to the multiple pathways and multiple levels of network pharmacology[14-15]. Notopterygii Rhizoma et Radix (NRR), as an important constituent of traditional Chinese medicine [16], derived from the dried roots and rhizomes of Notopterygium incisum Ting ex H. T. Chang (N. incisum) and Notopterygium franchetii H. de Boiss. (N. franchetii), belonging to Apiaceae [17]. Many experiments showed that NRR can resist arrhythmia[18-20], however, its mechanisms is still unclear. Here, we use various publicly available bioinformatics resources to investigate the potential pharmacological mechanisms of NRR in arrhythmia treatment.

\section{Materials And Methods}

\subsection{Screening of Active Ingredients}

Traditional Chinese Medicine Systems Pharmacology (TCMSP, https://tcmspw.com/index.php) Database and Analysis Platform, SymMap (http://www.symmap.org/), the Encyclopedia of Traditional Chinese Medicine (ETCM, http://www.tcmip.cn/ETCM/index.php/Home/Index/) Databases were searched for collect the chemical compounds of NRR [21-23]. The effective compounds filtered based on the pharmacokinetic properties, including oral bioavailability $(\mathrm{OB}) \geq 30 \%$ and drug-likeness (DL) index $\geq 0.18$. Furthermore, Two/Three-dimensional (2/3D) structure, canonical smiles and PubChem ID of the active compounds were acquired using PubChem (https://pubchem.ncbi.nlm.nih.gov/) database. The resulting data was standardized via Universal Protein Database (UniProt, https://www.uniprot.org/) database.

\subsection{Identification of Arrhythmia-associated Targets and Collection of Putative Target Proteins}

Comparative Toxicogenomics Database (CTD, http://ctdbase.org/)[24] and GeneCards (https://www.gene cards.org/)[25] databases were applied to identify potential targets related to arrhythmia, including ventricular premature beat (VPB), VT and ventricular fibrillation(Vf). Arrhythmia-associated genes were screened using scores $>50 \%$ of CTD and $30 \%$ of GeneCards as cutoffs, respectively. The candidate targets of NRR and arrhythmia were further used and pooled for Venn analysis to obtain the targets of NRR-achieved anti-arrhythmia.

\subsection{Constrction of Protein-Protien Interaction (PPI) Network and Topological Analysis}

The merged targets of NRR and arrhythmia were used to construct a PPI network via search tool for recurring instances of neighbouring genes (STRING) database (v11.5) (https://www.string-db.org/) with the active interaction sources from textmining, experiments, databases, co-expression, neighborhood and gene fusion, and the raw data saved as tab separated values (TSV) format. The AnalyzeNetwork tool of Cytoscape 3.8.2 was used to calculate the topological parameter [26]. The plug-in-MCODE (molecular 
complex detection) was used for module division; and CytoHubba was applied to detect the core targets based on the maximal cluque centrality (MCC) algorithm.

\section{$2.4 \mathrm{GO}$ and KEGG Enrichment}

The GO (Gene Ontology) and KEGG (Kyoto Encyclopedia of Genes and Genomes) enrichment analysis of ovelapping target and core targets were carried out through the Database for Annotation, Visualization and Integrated Discovery (DAVID) v6.8 (https://david.abcc.ncifcrf.gov/) [27-28] with the setting as follows: backgroud and species $=$ Homo sapiens, expression analysis systematic explorer $=0.01$ and count $=2$. $P<0.05$ indicates statistically significant difference in the annotation categories.

\subsection{Molecular Docking}

Small molecule ligand 2D structures of key active compounds obtained from PubChem (https://pubchem.ncbi.nlm.nih.gov) and saved in SDF format, and then, converted to 3D structure using ChemOffice. Crystal structures of core receptors were downloaded from protein data bank (PDB, https://www.rcsb.org/), removing ligand and water motifs with Pymol (v2.0), adding hydrogen with AutoDockTools (v1.5.6). AutoDock-Vina software was used to molecular docking, binding affinity calculation and probable binding sites locating.

\subsection{Experimental Validation}

\subsubsection{Experimental model and drug treatment}

NRR granula was purchased from the Granule Dispensing Department of Dongzhimen Hospital, Beijing University of Chinese Medicine. The calcium chloride was purchased from Sino Animal(BeiJing)Science and Technology Development Co. Ltd (Beijing, China).

The experimental procedures used in the present study were approved by the Institutional Animal Care and Use Committee from the Beijing Hospital of Traditional Chinese Medicine and were in accordance with the US National Institutes of Health (NIH Publications No.8023). Eighteen 8-week-old SD male rats were obtained from SPF (Beijing) Biotechnology Co., Ltd. [experimental animal license No. SYXK (Beijing), 2020-0051]. The rats were placed in polycarbonate cages under a 12-h light/dark cycle in an airconditioned room under a constant temperature $\left(25 \pm 1^{\circ} \mathrm{C}\right)$ and humidity $(50 \pm 10 \%)$ with grade SPF and were randomly divided into the following three groups: sham group (deionized water), vehicle group (deionized water), NRR group (NRR granule, $19.08 \mathrm{mg} / 100 \mathrm{~g}, 0.5 \mathrm{ml} / 100 \mathrm{~g}$ ). After gavage for 14 days, the rats were anesthetize 30 minutes after intragastric administration on the 14th day (6\% chloral hydrate, $0.5 \mathrm{ml} / 100 \mathrm{~g}$, intraperitoneally), and then linked electrocardiograph (ECG) lead. The $\otimes$ lead electrocardiogram was recorded using the PowerLab biological signal processing system. After the electrocardiogram was stable, the sham group was injected with saline $(0.9 \% \mathrm{NaCl}, 1.5 \mathrm{ml} / \mathrm{min} / \mathrm{kg})$ through the tail vein, the vehicle group and NRR groups were injected with calcium chloride solution $(1.5 \mathrm{ml} / \mathrm{min} / \mathrm{kg}$, concentration $35 \mathrm{mg} / \mathrm{ml})$ through the tail vein. The electrocardiogram was recorded for 10 
min, and the occurrence of arrhythmia was observed. The left cardiac myocardium (left ventricle) was frozen to analysis.

\subsubsection{Quantitative real-time PCR}

Five rats from each group were used for qRT-PCR. Total RNA was extracted from the left cardiac myocardium of the rats using TRIzol (Tiangen Biochemical Technology, Beijing, Co., Ltd) and reverse transcribed into cDNA using a reverse transcription system kit (Takara, Shanghai, China) according to the manufacturer's protocol. qRT-PCR was performed on an ABI 7500 Real-Time PCR System (Applied Biosystems, Foster City, USA) using the SYBR Green PCR Kit to determine mRNA expression levels. The relative expression of CASP3 (primers: GCGGTATTGAGACAGACAGTGGA and ACGAGTGAGGATGTGCATGAATT), CASP8 (primers: TTTCTGTTTTGGATGAGGTGAC and ATGTTGCTGAGTTTGGGTATGT), CASP9 (primers: GCGACATGATCGAGGATATTC and CAGGTGGTCTAGGGGTGTAAC), BAX (primers: GGTGGTTGCCCTTTTCTACTTTGC and GCTCCCGGAGGAAGTCCAGTG)\CDK2 (primers: CCCTGTCCGTACTTACACTCAT and CTTGGGGAAACTTGGCTTATA) was analyzed using the comparative CT method for relative quantitation and the 2-DDCt method by normalizing to b-actin expression, and relative expression is presented as the percent change compared to matched controls. All quantitative data, obtained from at least three independent experiments, are presented as the mean \pm standard error of the mean. SPSS statistical software version 27.0 (IBM Corp., Armonk, NY, USA) was used for statistical analysis. Variance between two groups were compared by Student's t-test, and multiple groups were compared by one-way analysis of variance. $P$ values less than 0.05 was considered statistically significant.

\section{Results}

\subsection{Active Compounds and Target Genes Detection}

Details on revealing the pharmacological mechanisms of NRR against arrhythmia are shown in Figure 1. There were 185, 235 and 58 components obtained from TCMSP, SymMap and ETCM database, respectively. After screening by pharmacokinetic properties and removaling duplicate, 21 active components were included, dividing into 5 categories: 15 coumarin (e.g., Cnidilin, Ammidin, Phellopterin, etc.), 1 flavonoid (Diosmetin), 1 nitrogen-containing compound (Isoindigo), 2 triterpenoids (beta-sitosterol and Sitosterol) and 2 others (Figure 2). The details of 21 compounds see in table S1. Then, the targets were predicted by UniProt database, and a total of 159 putative targets were identified, including 105 in coumarin, 10 in flavonoid, 3 in nitrogen-containing compound, 40 in triterpenoids and 1 in others. A compound-target network was constructed as shown in Figure 3. Eventrally, 57 targets of NRR against arrhythmia were obtained after the removal of repetitions.

\subsection{Putative NRR Target Proteins in Arrhythmia}

There were 96342 and 5584 arrhythmia-associated gene acquired from CTD and GeneCards databased, respectively. With prioritized inference and relevance scores, 452 and 97 genes were identified (Table 
S3\&S4). By integrating the data from the two databases, 265 targets were included and considered as the key putative arrhythmia-associated proteins. As shown in the Venn diagram, a total of 19 overlapping genes of NRR and arrhythmia were obtained (Figure 4A), including CDK2, BAX, CASP3, etc., which were taken as the key targets for the treatment of arrhythmia with NRR.

\subsection{PPI Construction and Hub Genes Identification}

A function-associated protein network of the interaction between NRR and arrhythmia was constructed, which included 19 nodes and 40 edges (Figure 4B). The topological parameters were analyzed based on Analyze Network (Table S5). Only one module was divided through MCODE, which considered as the core module (Figure 4C). Futhermore the core proteins were identified via CytoHubba, including CASP3 (caspase 3), CASP8 (caspase 8), CASP9 (caspase 9), CDK2 (Cell division protein kinase 2) and BAX (Apoptosis regulator BAX); and these core targets all belonged to the core module (Figure 4D).

\subsection{Biological Function of NRR Targeting on Arrhythmia}

To perform further insight into the mechanisms underlying NRR effects on arrhythmia at the systematic level, GO and KEGG pathway analyses were carried out. There were $141 \mathrm{GO}$ terms and $31 \mathrm{KEGG}$ pathways enriched from the 19 overlapping targets (Tables S6\&S8), and 33 GO terms and 16 KEGG pathways enriched from 5 core targets (Tables S7\&S9). The GO-biological process mainly related to response to drug, response to lipopolysaccharide, response to estradiol, extrinsic apoptotic signaling pathway in absence of ligand, apoptotic process etc. Among the pathways enriched by overlapping targets and core targets, 15 are consistent. The top 3 pathways enriched by 5 core targets were p53 signaling pathway, Hepatitis B and Apoptosis. A drug-compound-targets-pathways network was builded base on the KEGG enrichment analysis (Figure 5).

\subsection{Molecular Docking}

The affinity between the two counterparts can be predicted by calculating the binding energy. Ten active NRR componds with good pharmacokinetic properties were molecularly docked with 5 core receptors, including CASP3 (PDBID: 1NME), CASP8 (PDBID: 2K7Z), CASP9 (PDBID: 3V3K), BAX (PDBID: 2LR1), CDK2 (PDBID: 2R3J). The binding energy of less than 0 meaned that two molecules combine spontaneously. The lower the binding energy, the stronger the affinity of the compounds to the targets. A total of 50 ligand-receptor combinations were computed. The majority of NRR components bind well with the key receptors, in which 44 combinations had affinities of $<-7 \mathrm{kcal} / \mathrm{mol}$, account for $88 \%$. CDK2 and C9 had the strongest binding energy, at $-9.7 \mathrm{kcal} / \mathrm{mol}$. The molecular binding findings are shown in Fig. 6 .

\subsection{Experimental Validation on the Targets of NRR in Treating Arrhythmia}

The ECG of sham, vehicle and NRR-treated group were showed in Fig. 8. CASP3, CASP8, CASP9, BAX and CDK2 mRNA expression were consistently altered in individual samples from the sham, vehicle and NRR 
groups. The expression of CASP3, CASP8, CASP9, BAX and CDK2 were upregulated after arrhythmia, while the CASP3, CASP8, CASP9, BAX and CDK2 expression were downregulated after treated with NRR for 14 days.

\section{Discussion}

In this study, a network pharmacology analysis was conducted on the active components of NRR in arrhythmia disease targets. The ingredients contained in NRR mainly included coumarin, flavonoids, nitrogen-containing compounds, and triterpenoids, among which coumarin was the primary category. The results were accordance with the detection of ultra high performance liquid chromatography coupled with triple quadrupole mass spectrometry(UHPLC-MS/MS)[29]. Previous studies also verified the aqueous solution of NRR has anti-arrhythmic effect[30-31]. Biological processes of NRR protein associated with arrhythmia were involved in various apoptotic processes, such as endopeptidase activity involved in apoptotic process by cytochrome c, extrinsic apoptotic signaling pathway in absence of ligand. Moreover, p53 signaling pathway may closely associate with mechanisms of action of NRR proteins related to arrhythmia.

For further investigation, we conduct module division and find th core genes by MCC in our work. CASP3, CASP8, CASP9, BAX and CDK2 were identified from the module involved in p53 signaling. In addition, the results of molecular docking also exhibited that all of them have good binding properties with core target genes. Clinical studies have shown that the expression of BAX was increased in cardiovascular patients, including arrhythmia[32], and caspase3 variants was susceptible to atrial fibrillation (AF). Myocardial apoptosis can cause electrical and structural cardiac remodeling[33]; and as the important apoptotic factors, the expression of CASP8, CASP9, BAX was altered in many cardiovascular disease (e.g. ichaemia/reperfusion[34], AF[35]). Oxidative stress can cause apoptosis, which in turn leads to cardiac and vascular abnormalities in different types of cardiovascular diseases, and its mechanism may be related to the dysregulation of Akt/p53 signaling pathway[36]. In summary, our findings offer new insights on future NRR research and its applications in arrhythmia treatment. However, apoptosis is a complicated process regulated by multitargets. Although our results were verified by qRT-PCR, different componets and doses will be done in future study to find more evidence.

\section{Conclusion}

Here, we applied systematic pharmacology to investigate the potential mechanisms underlying NRR effects on arrhythmia. The analysis of modules and core nodes help us locate the possible major functional genes. Our data show that NRR, especially the main category (coumarin and triterpenoids), plays an anti-apoptotic role via multilple apoptotic factors. The targets mainly targets the downstream of the p53 signaling pathways, which may be its main mechanism against arrhythmia.

\section{Abbreviations}


PVCs premature ventricular contractions

VT ventricular tachycardia

TCM Traditional Chinese Medicine

NRR Notopterygii Rhizoma Et Radix

TCMSP Traditional Chinese Medicine Systems Pharmacology

ETCM Encyclopedia of Traditional Chinese Medicine

OB oral bioavailability

DL drug-likeness

CTD Comparative Toxicogenomics Database

VPB ventricular premature beat

Vf ventricular fibrillation

PPI Protein-Protien Interaction

STRING search tool for recurring instances of neighbouring genes

MCODE molecular complex detection

MCC maximal cluque centrality

GO Gene Ontology

KEGG Kyoto Encyclopedia of Genes and Genomes

DAVID Database for Annotation, Visualization and Integrated Discovery

PDB protein data bank

ECG electrocardiograph

QT-PCR Quantitative real-time Polymerase Chain Reaction

UHPLC-MS/MS ultra high performance liquid chromatography coupled with triple quadrupole mass spectrometry

AF atrial fibrillation 


\section{Declarations}

\section{Ethics approval and consent to participate}

Not applicable.

\section{Consent for publication}

Not applicable.

\section{Availability of data and materials}

The datasets used and/or analysed during the current study are available from the corresponding author on reasonable request.

\section{Competing interests}

The authors declare that they have no competing interests.

\section{Funding}

This work was supported by Grants from the National Natural Science Foundation of China (No. 81273741) and the National Administration of Traditional Chinese Medicine (NO. 2019XZZX-XXG001).

\section{Authors' contributions}

Hongxu Liu and Juju Shang contributed to the conception or design of the work. Xiang Li and Wen long Xing revised the manuscript; Dehuai Long and Yupei Tan is responsible to data collection; Yifei Qi is responsible to data reduction and analysis, and Penglu Wei drafted the manuscript. The final contribution was read and approved by all the authors.

\section{Acknowledgements}

Not applicable.

\section{References}

1. Nguyen HL, Vaseghi M. Sympathetic Denervation for Treatment of Ventricular Arrhythmias. J Atr Fibrillation. 2020;13(1):2404. doi: 10.4022/jafib.2404.

2. Markman TM, Nazarian S. Treatment of Ventricular Arrhythmias: What's New?.Trends Cardiovasc Med.2018:29(5):249-261. doi: 10.1016/j.tcm.2018.09.014.

3. Dresen WF, Ferguson JD. Ventricular Arrhythmias. Cardiol Clin.2018;36(1):129-139. doi: 10.1016/j.ccl.2017.08.007. 
4. Grace AA, Dan MR. Systems biology and cardiac arrhythmias. Lancet. 2012;380(9852):1498-1580. doi:10.1016/S0140-6736(12)61462-7.

5. Dow LE, Lowe SW. Life in the fast lane: mammalian disease models in the genomics era. Cell. 2012;148(6):1099-109. doi: 10.1016/j.cell.2012.02.023.

6. Cherry ABC, Daley GQ. Reprogramming cellular identity for regenerative medicine. Cell. 2012;148(6):1110-22. doi: 10.1016/j.cell.2012.02.031.

7. Magnani JW, Rienstra $\mathrm{M}$, Lin $\mathrm{H}$, et al. Atrial fibrillation: current knowledge and future directions in epidemiology and genomics. Circulation. 2011;124(18):1982-93.

doi:10.1161/CIRCULATIONAHA.111.039677.

8. Kathiresan S, Srivastava D. Genetics of human cardiovascular disease. Cell. 2012;148(6):1242-57. doi: 10.1016/j.cell.2012.03.001.

9. Zhou Q, Xiao J, Jiang D, et al. Carvedilol and its new analogs suppress arrhythmogenic store overload-induced Ca2+ release. Nat Med. 2011;17(8):1003-9. doi: 10.1038/nm.2406.

10. Jung CB, Moretti A, Mederos YSM, et al. Dantrolene rescues arrhythmogenic RYR2 defect in a patientspecific stem cell model of catecholaminergic polymorphic ventricular tachycardia. EMBO Mol Med.2012;4(3):180-91. doi: 10.1002/emmm.201100194.

11. Luo TT, Lu Y, Yan SK, et al. Network Pharmacology in Research of Chinese Medicine Formula: Methodology, Application and Prospective. Chin J Integr Med. 2019;26(1):72-80. doi: 10.1007/s11655-019-3064-0.

12. Boezio B, Audouze K, Ducrot P, et al. Network-based Approaches in Pharmacology. Mol Inform. 2017;36(10). doi: 10.1002/minf.201700048.

13. Zhang RZ, Zhu X, Bai H, et al. Network Pharmacology Databases for Traditional Chinese Medicine: Review and Assessment. Front Pharmacol. 2019;10:123. doi: 10.3389/fphar.2019.00123

14. Wei M, Li H, Li Q, et al. Based on Network Pharmacology to Explore the Molecular Targets and Mechanisms of Gegen Qinlian Decoction for the Treatment of Ulcerative Colitis. Biomed Res Int.2020;2020:5217405. doi: 10.1155/2020/5217405.

15. Xu J, Wang F, Guo J, et al. Pharmacological Mechanisms Underlying the Neuroprotective Effects of Alpinia oxyphylla Miq. on Alzheimer's Disease. Int J Mol Sci. 2020;21(6):2071. doi: 10.3390/ijms21062071.

16. Chinese Pharmacopoeia Commission, Pharmacopoeia of the People's Republic of China. China Medical Science and Technology Press, Beijing, 2015;182-183.

17. Hong L, Juanjuan Z, Xiaoxiang Z, et al. Therapeutical effect of Radix Aconiti and Astragalus extracts on models of experimental bradycardia animal.Pak J Pharm Sci. 2014;27(3):439-44. doi:10.1016/j.drudis.2014.01.001

18. Guo P, Lang YJ, Zhang GT. Research progress on chemical constituents and pharmacological activities of Notopterygii Rhizoma et Radix. Chin Tradit Pat Med. 2019;41(10):2445-2459. doi: 
19. Gong ZH, Duan YQ, Fu XY, et al. Study on pharmacological mechanisms of Notopterygii Rhizoma et Radix. Asia-Pacific Tradit Med. 2019;15(5): 192-194. doi:10.11954/ytctyy.201905061

20. Ma LM, Yang JL. Research progress on chemical constituents and pharmacological activities of Notopterygii Rhizoma et Radix. Chin Tradit Herbal Drugs. 2021;52(19):6111-6120. doi:10.7501/j.issn.0253-2670.2021.19.034

21. Jin LR, Peng L, Ji NW, et al. TCMSP: a database of systems pharmacology for drug discovery from herbal medicines. J Cheminform. 2014;6:13. doi: 10.1186/1758-2946-6-13.

22. Wu Y, Zhang F, Yang K, et al. SymMap: an integrative database of traditional Chinese medicine enhanced by symptom mapping. Nucleic Acids Res. 2019;47(D1):D1110-D1117. doi: 10.1093/nar/gky1021.

23. Xu HY, Zhang YQ, Liu ZM, et al. ETCM: an encyclopaedia of traditional Chinese medicine. Nucleic Acids Res. 2019;47(D1):D976-D982. doi: 10.1093/nar/gky987.

24. AP Davis, CJ Grondin, RJ Johnson, et al. The Comparative Toxicogenomics Database: Update 2019. Nucleic Acids Res. 2019;47(D1):D948-D954. doi: 10.1093/nar/gky868.

25. G Stelzer, N Rosen, I Plaschkes, et al. Plaschkes et al. The GeneCards Suite: From Gene Data Mining to Disease Genome Sequence Analyses. Curr Protoc Bioinformatics. 2016;54:1.30.1-1.30.33. doi: 10.1002/cpbi.5.

26. P Shannon, A Markiel, O Ozieret, et al. Cytoscape: A software environment for integrated models of biomolecular interaction networks. Genome Res. 2003;13(11):2498-504. doi: 10.1101/gr.1239303.

27. Huang DW, Sherman BT, Lempicki RA. Systematic and integrative analysis of large gene lists using DAVID Bioinformatics Resources. Nat Protoc. 2009;4(1):44-57. doi: 10.1038/nprot.2008.211.

28. Huang DW, Sherman BT, Lempicki RA. Bioinformatics enrichment tools: paths toward the comprehensive functional analysis of large gene lists. Nucleic Acids Res. 2009;37(1):1-13. doi: 10.1093/nar/gkn923.

29. Wu XW, Zhang YB, Lei Z, et al. Simultaneous quantification of 33 active components in Notopterygii Rhizoma et Radix using ultra high performance liquid chromatography with tandem mass spectrometry. J Chromatogr B Analyt Technol Biomed Life Sci. 2018;1092:244-251. doi: 10.1016/j.jchromb.2018.06.006.

30. Ma YL, Lin JM, Ma L, et al. Progress in Researches on Notopterygium. J Anhui Agricultural Sci. 2010;38(24):13092-13093. doi:10.13989/j.cnki.0517-6611.2010.24.095

31. Cheng YZ, Shan ZY, Chen YP. Comparison of antiarrhythmic effects of different components of Qianghuishui solution. J Basic Chin Med. 1998;4(2):43.

32. Asma M, Ali RB, Vahid DM, et al. Evaluation of Oxidative Stress, Apoptosis, and Expression of MicroRNA-208a and MicroRNA-1 in Cardiovascular Patients. Rep Biochem Mol Biol. 2021;10(2): 183-196. doi: 10.52547/rbmb.10.2.183 
33. Zhan C, Liu G, Li J, et al. Rotenone and 3-bromopyruvate toxicity impacts electrical and structural cardiac remodeling in rats. Toxicol Lett. 2019;318:57-64. doi: 10.1016/j.toxlet.2019.09.024.

34. Zhang J, Zhang X. Ischaemic preconditioning-induced serum exosomes protect against myocardial ischaemia/reperfusion injury in rats by activating the PI3K/AKT signalling pathway. Cell Biochem Funct. 2020;39(2):287-295. doi: 10.1002/cbf.3578.

35. Luo ZR, Yan $\mathrm{CH}, \mathrm{Yu}$ P, et al. CASP3 genetic variants and susceptibility to atrial fibrillation in Chinese Han population. Int J Cardiol. 2015;183:1-5.doi: 10.1016/j.ijcard.2015.01.048.

36. Zheng N, Li H, Wang X, et al. Oxidative stress-induced cardiomyocyte apoptosis is associated with dysregulated Akt/p53 signaling pathway. J Recept Signal Transduct Res. 2020;40(6):599-604. doi: 10.1080/10799893.2020.1772297.

\section{Figures}

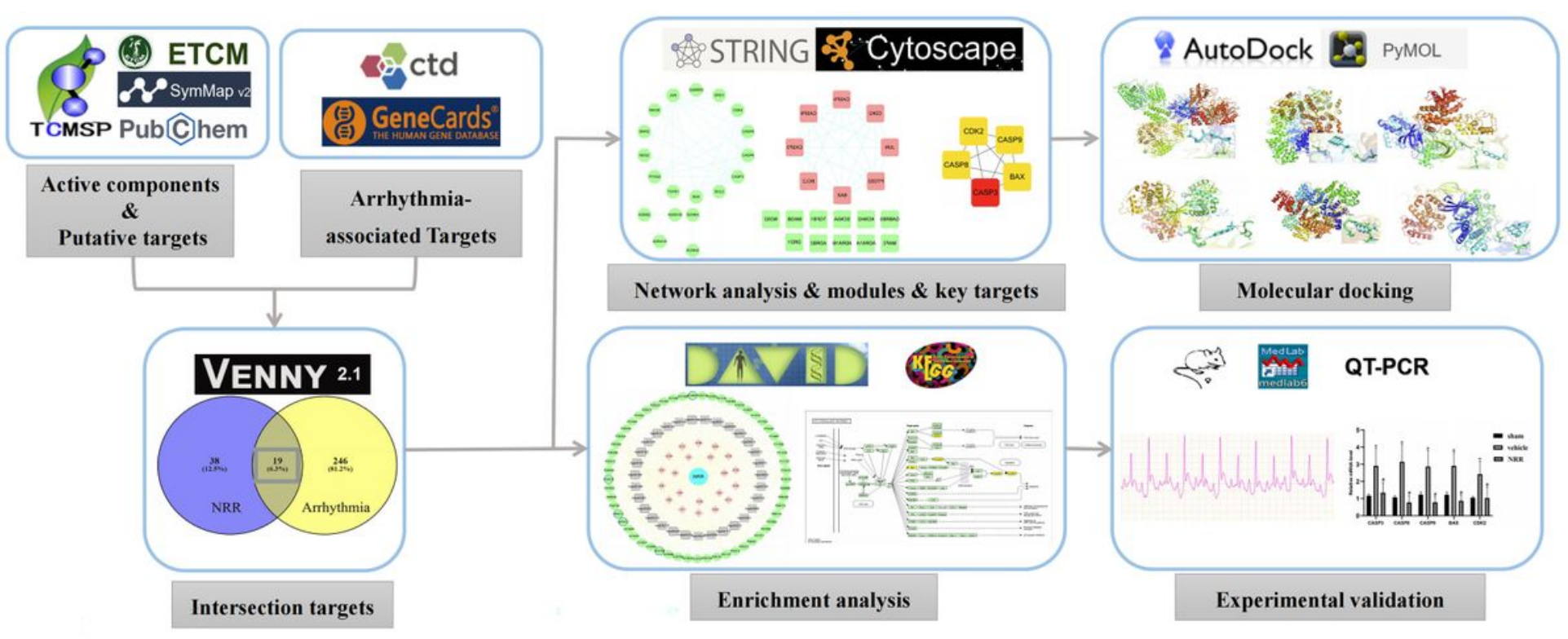

\section{Figure 1}

Network pharmacology for deciphering pharmacological mechanisms of NRR in treating arrhythmia. 

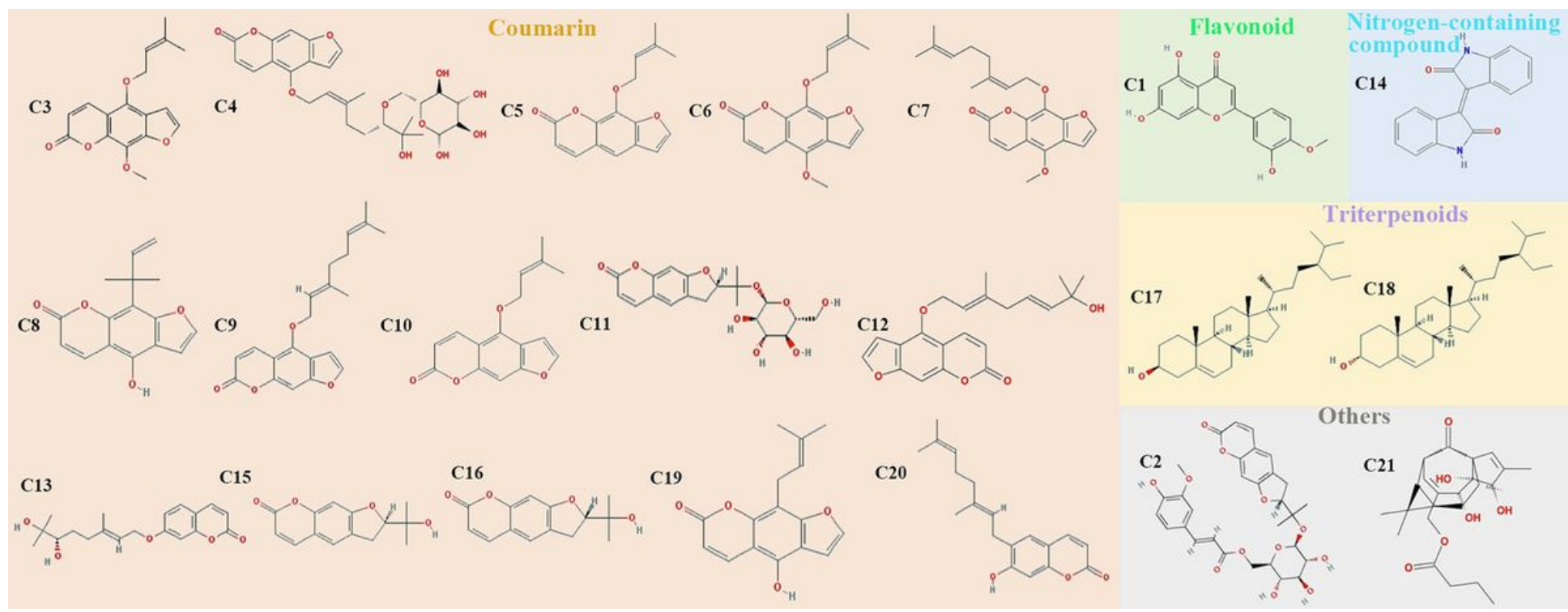

\section{Figure 2}

The 2-dimensional (2D) molecular structures and classification of 21 NRR candidate compounds. There were five categories including coumarin(15), triterpenoids(2), nitrogen-containing compound(1), flavonoid(1), others(2).

P19793 P11309

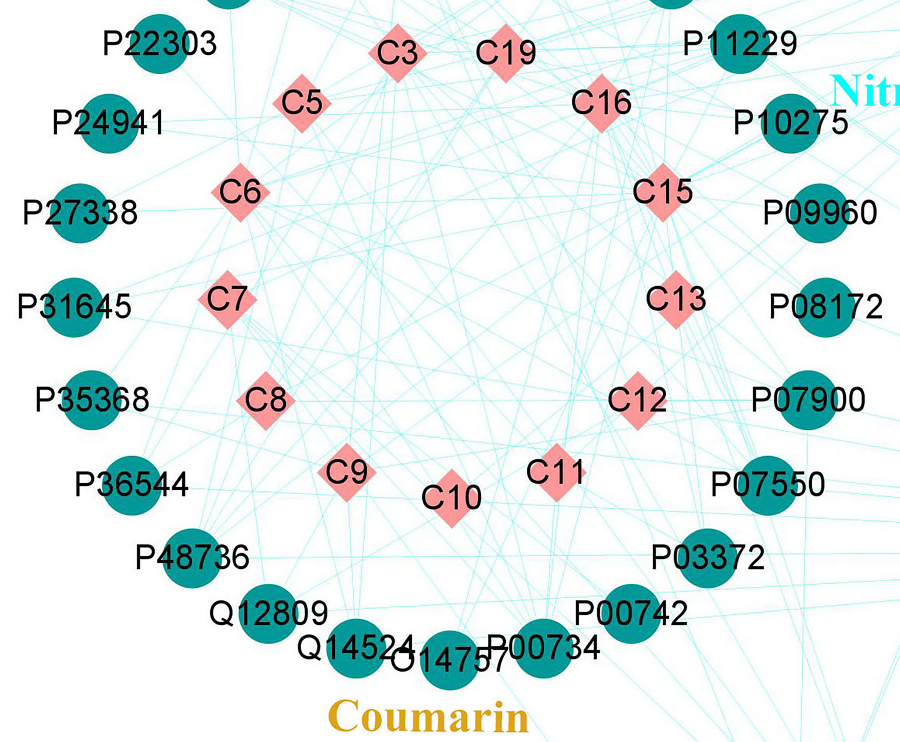

P48539 P35228

Q15788 $\begin{array}{ll}\text { C1 } & \\ & \text { P27487 }\end{array}$

P07477

Flavonoid

\section{Q14568 P35354}

C14

P23219

P08235 C21

Others

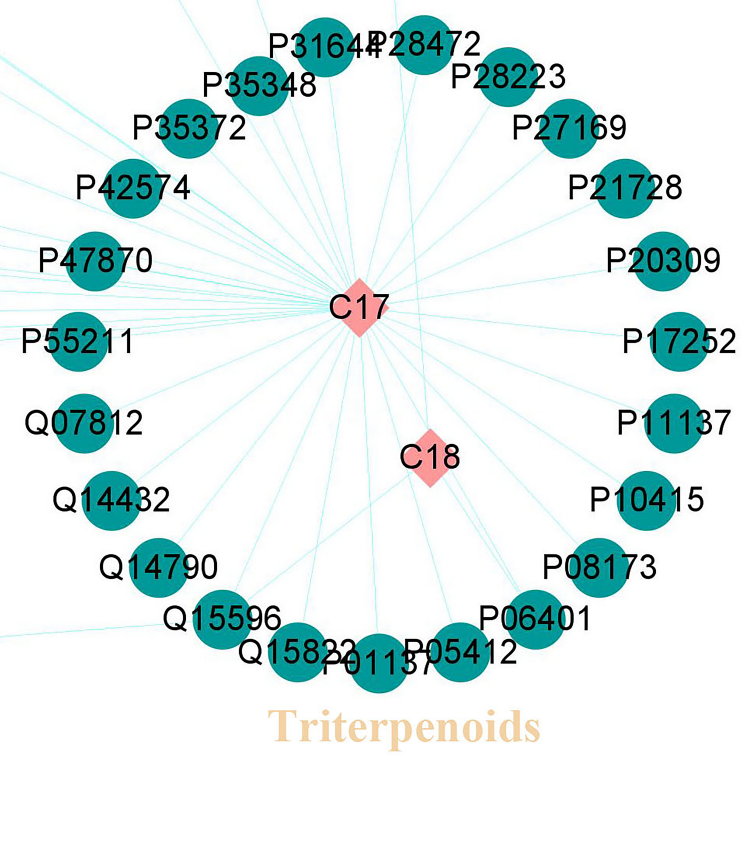




\section{Figure 3}

The construction of compound-target network, and the classification of putative target proteins of NRR compounds.

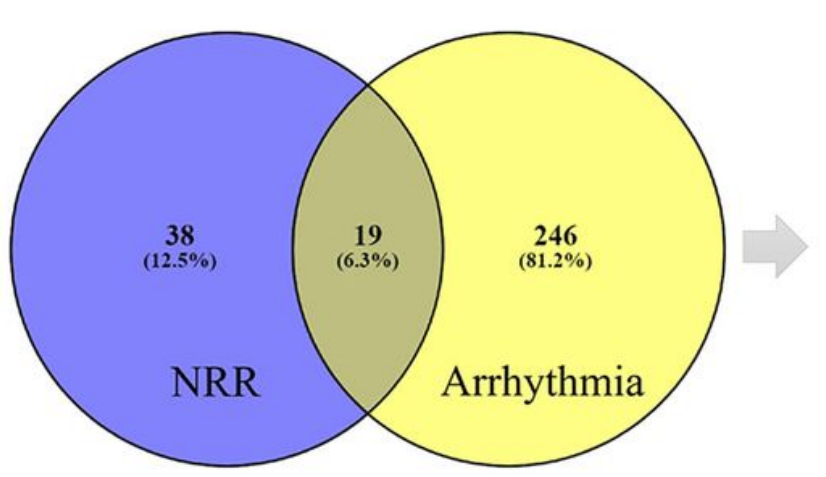

Overlapping targets

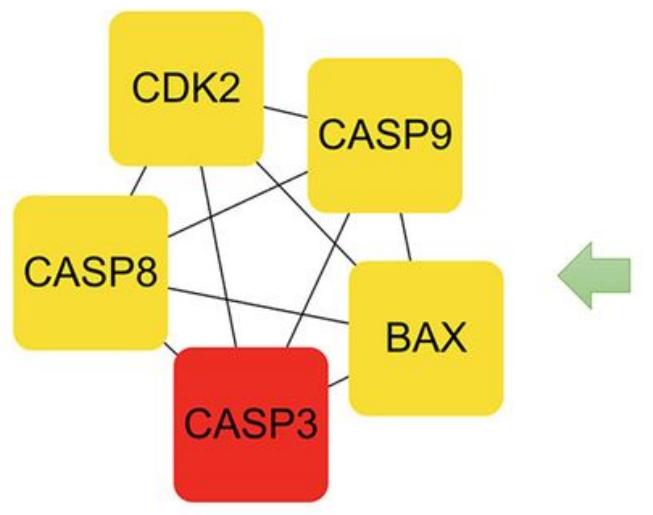

Key targets

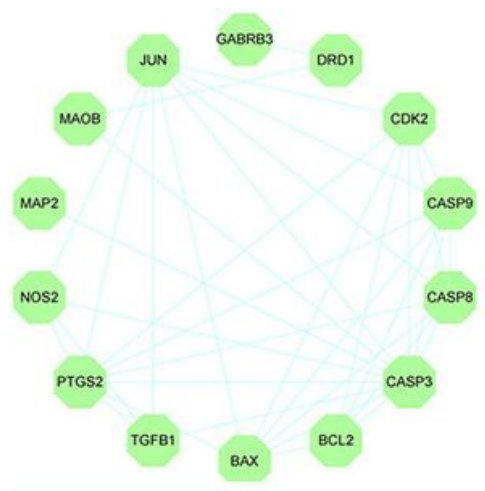

Network

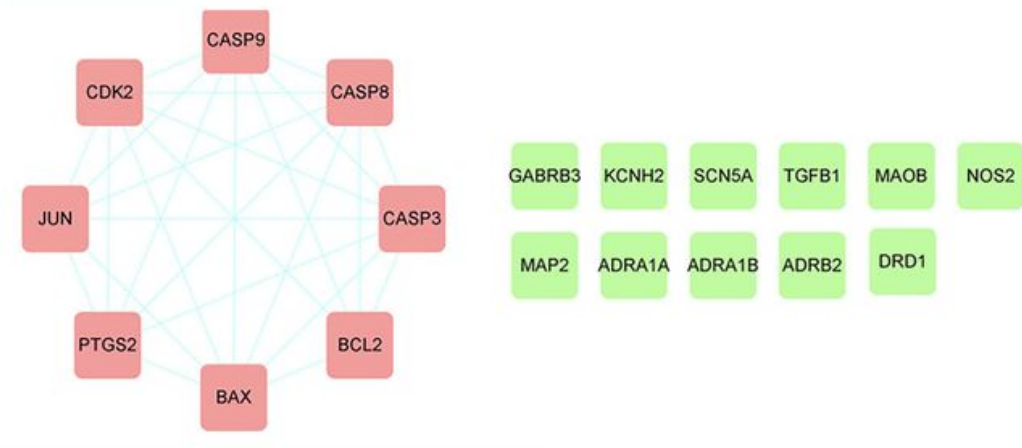

Module

\section{Figure 4}

The identified genes of NRR and arrhythmia and the construction of protein-protein interaction network. 


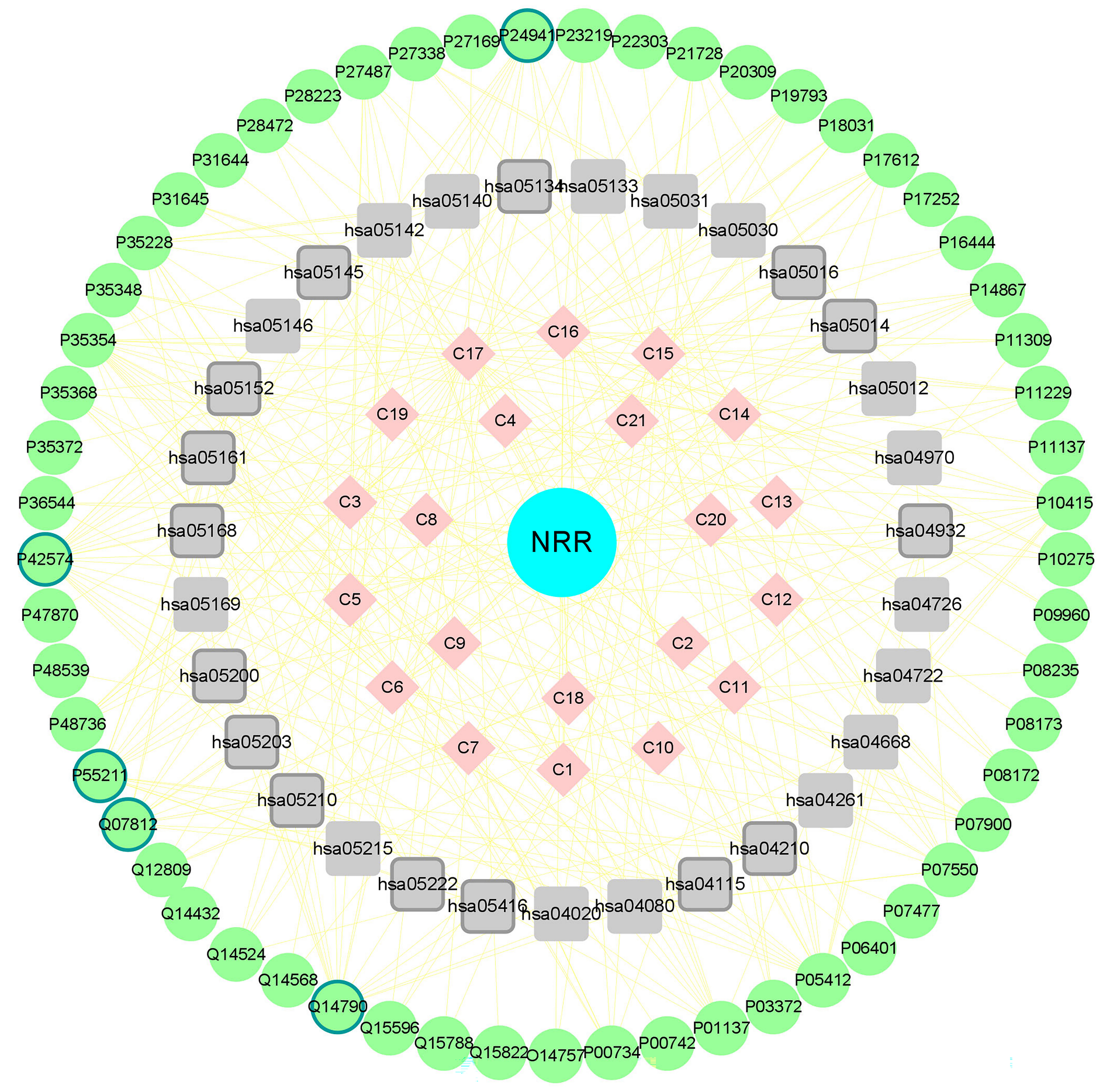

Figure 5

Construction of drug-compound-target-pathway network. (wathet circle represents the herb-NRR; pink diamond represents the compounds in NRR; green circle represents the targets, with green border reprsenting core targets; gray rectangle represents KEGG signaling enriched form 19 overlapping target proteins of NRR associated with arrhythmia, with gray border representing the KEGG pathways enriched from 5 core targets. 
A

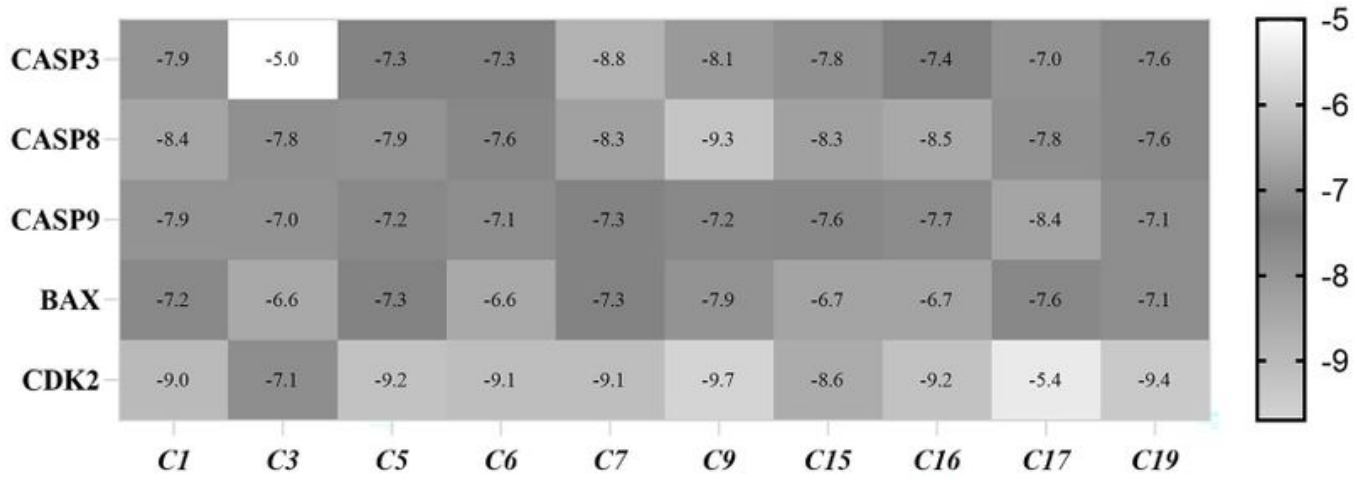

B

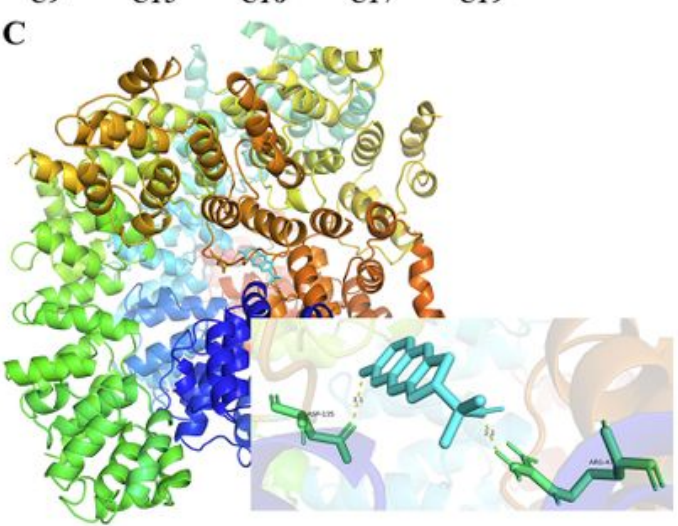

D

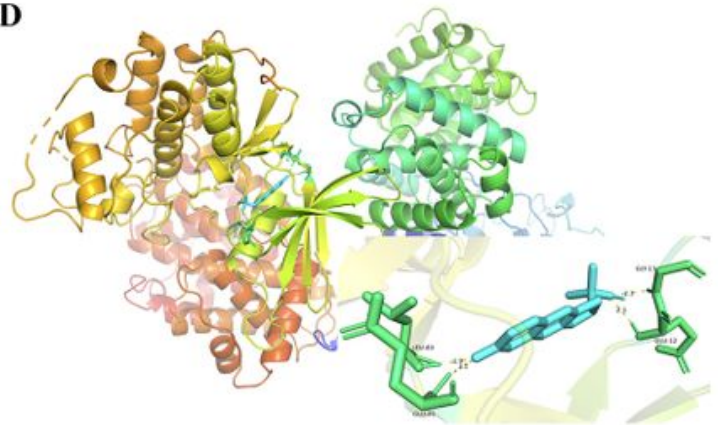

E

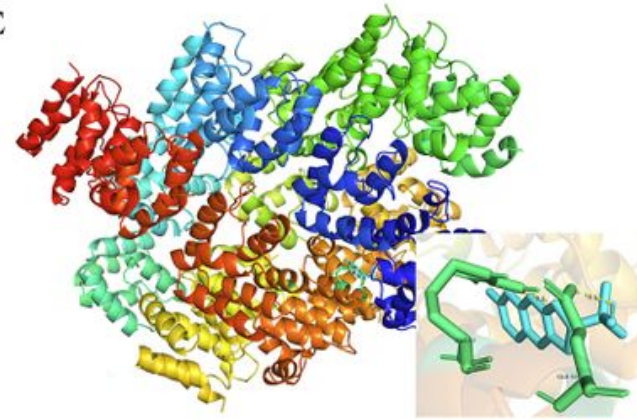

F

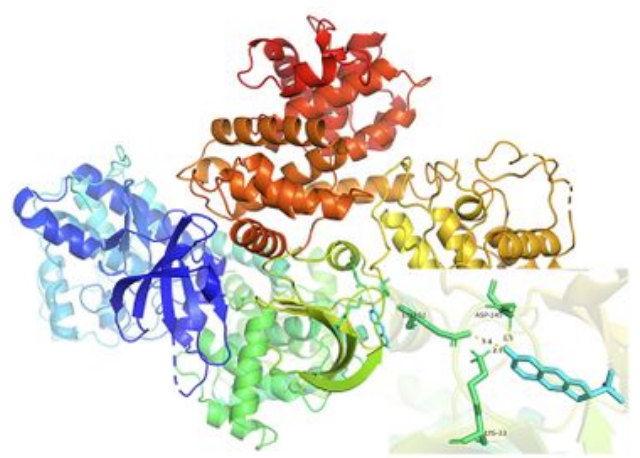

G

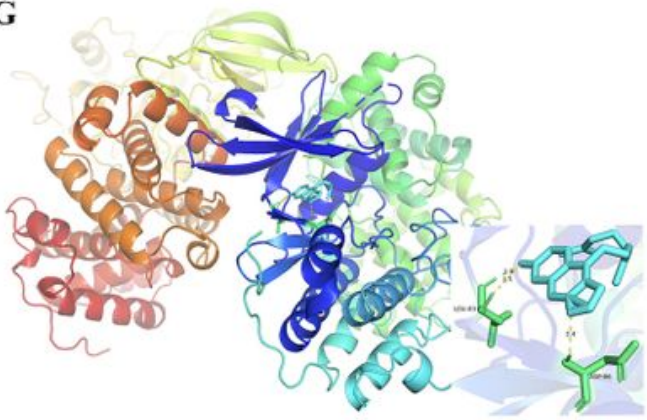

Figure 6

Results of molecular docking and docking simulation. A Heat map of the binding energies. B betasitosterol (C17) with CAS9 (PDBID: 3V3K), C Marmesine (C15) with CASP8 (PDBID: 2K7Z), D Marmesine (C15) with CDK2 (PDBID: 2R3J), E Marmesin (C16) with CASP8, F Marmesin (C16) with CDK2, and G Bergaptin (C9) with CDK2. 


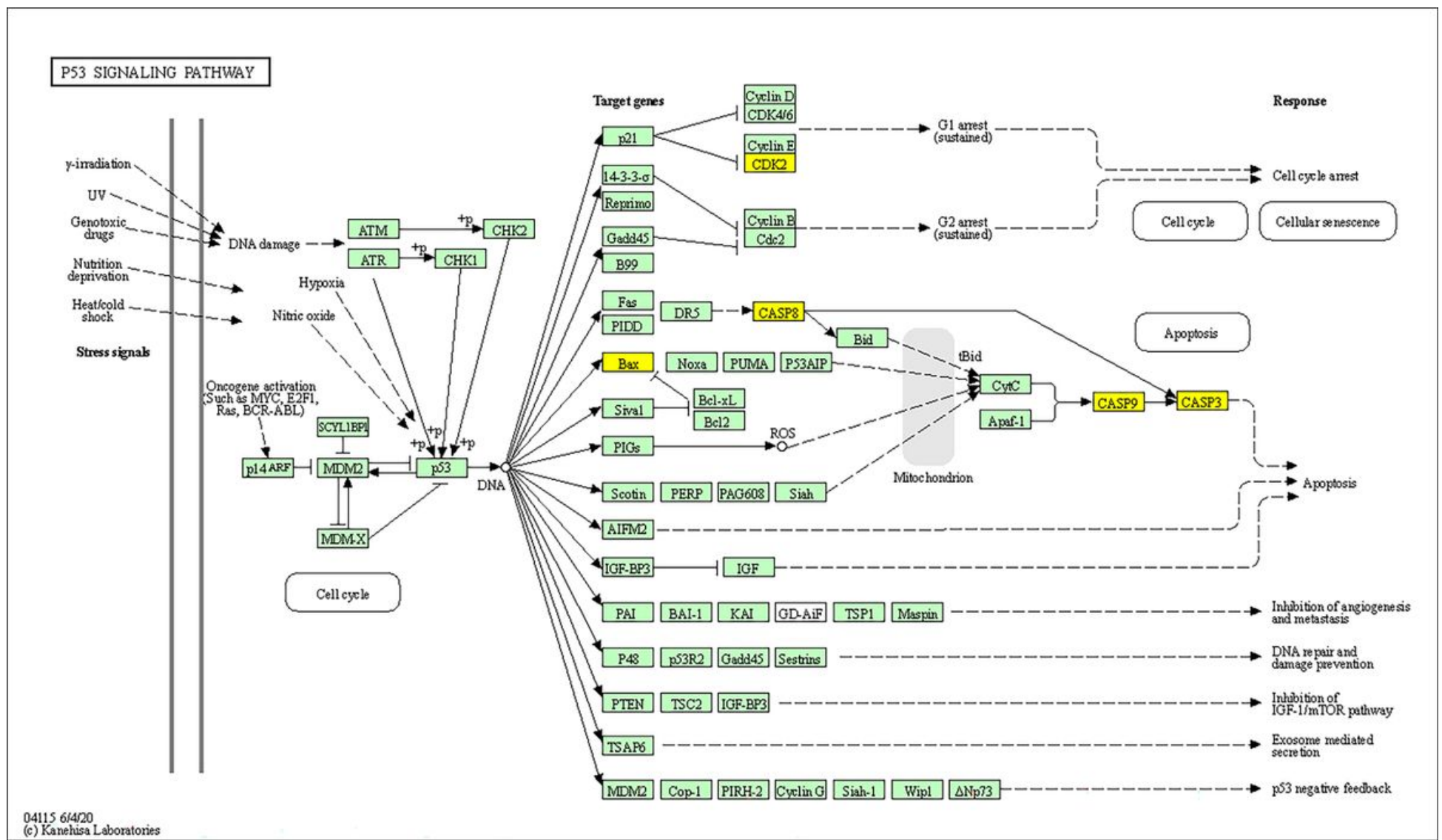

Figure 7

p53 signaling pathway (KEGG database). Core receptors are shown in yellow, and other protein targets are in green.

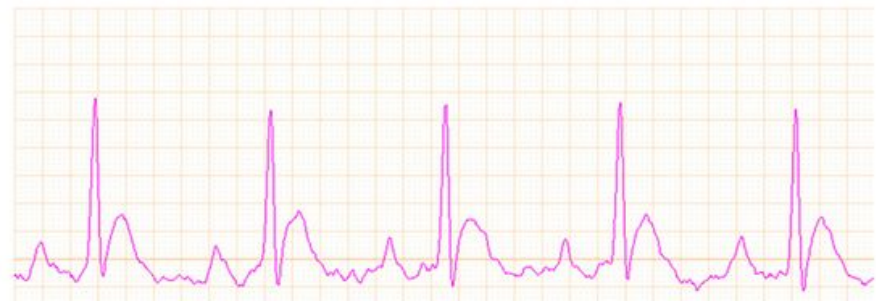

Sham

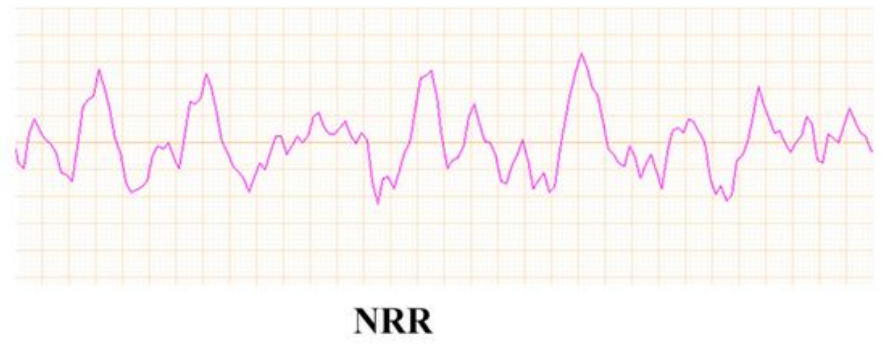

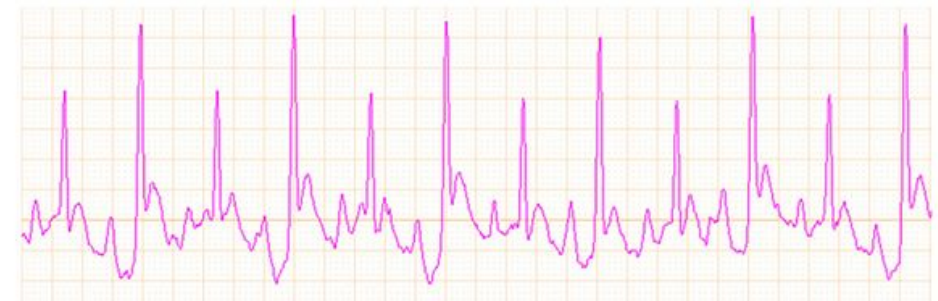

Vhicle

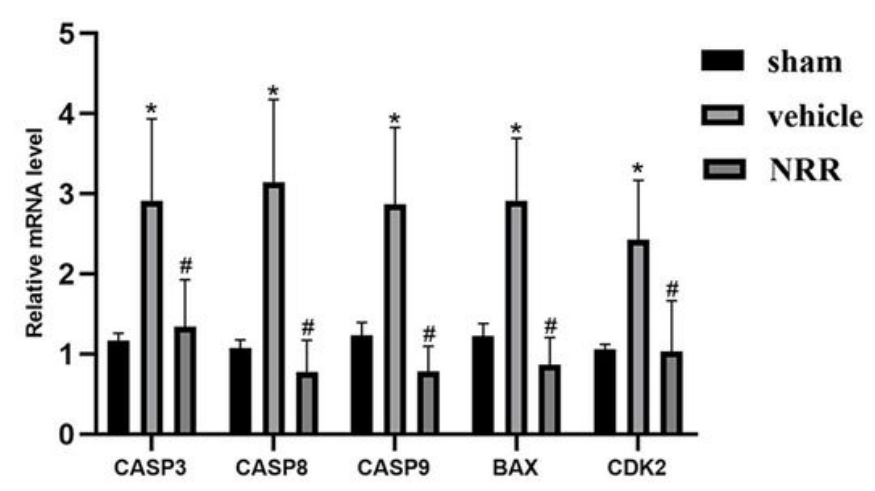


Figure 8

Validation by the mRNA expression levels of CASP3, CASP8, CASP9, BAX and CDK2 using real-time RTPCR. * $\mathrm{P}<0.05$.

\section{Supplementary Files}

This is a list of supplementary files associated with this preprint. Click to download.

- supplementarymaterials.docx 